\title{
Vasculo-Behçet's Disease that Began with Femoral Arterial Pseudoaneurysm Followed by Deep Venous Thrombosis: Report of a Case
}

\author{
Tomohiro Haruki, MD, Shigeto Miyasaka, MD, Hiroyuki Maeta, MD, Keisuke Morimoto, MD, \\ and Iwao Taniguchi, MD
}

\begin{abstract}
Vasculo-Behçet's disease mainly affects the venous system and central or peripheral arteries. It is often difficult to diagnosis this due to the complexity of symptoms and the rarity. A 35-year-old man with refractory inguinal lymphadenitis was admitted to our hospital. He was diagnosed with left femoral arterial pseudoaneurysm by enhanced CT scan of the lower legs, and we performed an emergency operation. Seven months postoperatively, he suddenly developed deep venous thrombosis, and then, symptoms such as aphthous stomatitis and pudendal ulcer started to develop progressively, complicating his uveitis. Finally, he was diagnosed with vasculo-Behçet's disease.
\end{abstract}

Key words: vasculo-Behçet's disease, femoral arterial pseudoaneurysm, deep venous thrombosis

\section{INTRODUCTION}

$\mathrm{B}$ ehçet's disease (BD) is a multi-systemic, chronic, inflammatory disorder characterized mainly by aphthous stomatitis, genital ulcers, skin lesions, and ocular manifestations. ${ }^{1)}$ Sometimes patients with BD develop vascular complications such as venous thrombosis, aneurysm formation, and arterial occlusion, by which they are diagnosed with vasculo-Behçet's disease (VBD). ${ }^{2)}$ The diagnosis of BD is based on clinical criteria consisting of combinations of symptoms because of the lack of universally recognized pathognomonic laboratory tests. Therefore, it is sometimes very difficult to diagnose BD definitively. Although vascular lesions often develop during the chronic phase of the disease, in our case, they were the earliest symptoms and other symptoms such as aphthous

Department of Thoracic and Cardiovascular Surgery, Tottori Prefectural Central Hospital, Tottori, Japan

Received: June 6, 2008 Accepted: November 4, 2009

Address for reprint requests to Tomohiro Haruki: Department of Thoracic and Cardiovascular Surgery, Tottori Prefectural Central Hospital, 730 Ezu, Tottori-City, Tottori 680-0901, Japan

Tel: +81-857-26-2271, Fax: +81-857-29-3227

E-mail: tomohiro19780703@yahoo.co.jp stomatitis and pudendal ulcer became apparent later. We describe a 35-year-old man in whom VBD began to develop with femoral arterial pseudoaneurysm and deep venous thrombosis, and we discuss the difficulty of diagnosis of this disease.

\section{Case Report}

A 35-year-old man was admitted to our hospital with a complaint of left inguinal mass. He had a history of unidentified fever that abated spontaneously. He had no history of previous trauma, fractures, or recent infections. He was a pack-a-day smoker and he was not on any medication. Physical examination revealed a non-pulsatile mass, about $10 \mathrm{~cm}$ in diameter, in the left femoral area and entire left lower limb swelling (Fig. 1A). Firstly, he was falsely diagnosed as acute suppurative lymphadenitis from a severe inflammatory reaction on blood examination and the findings of plain computed tomography (CT) scan of the lower leg (Fig. 1B), and was treated with antibiotics at the department of dermatology in our hospital. Although treatment with various antibiotics had continued over the course of a month, his left inguinal mass and lower limb swelling did not improve. Then, he consulted our department to receive surgical treatment for 

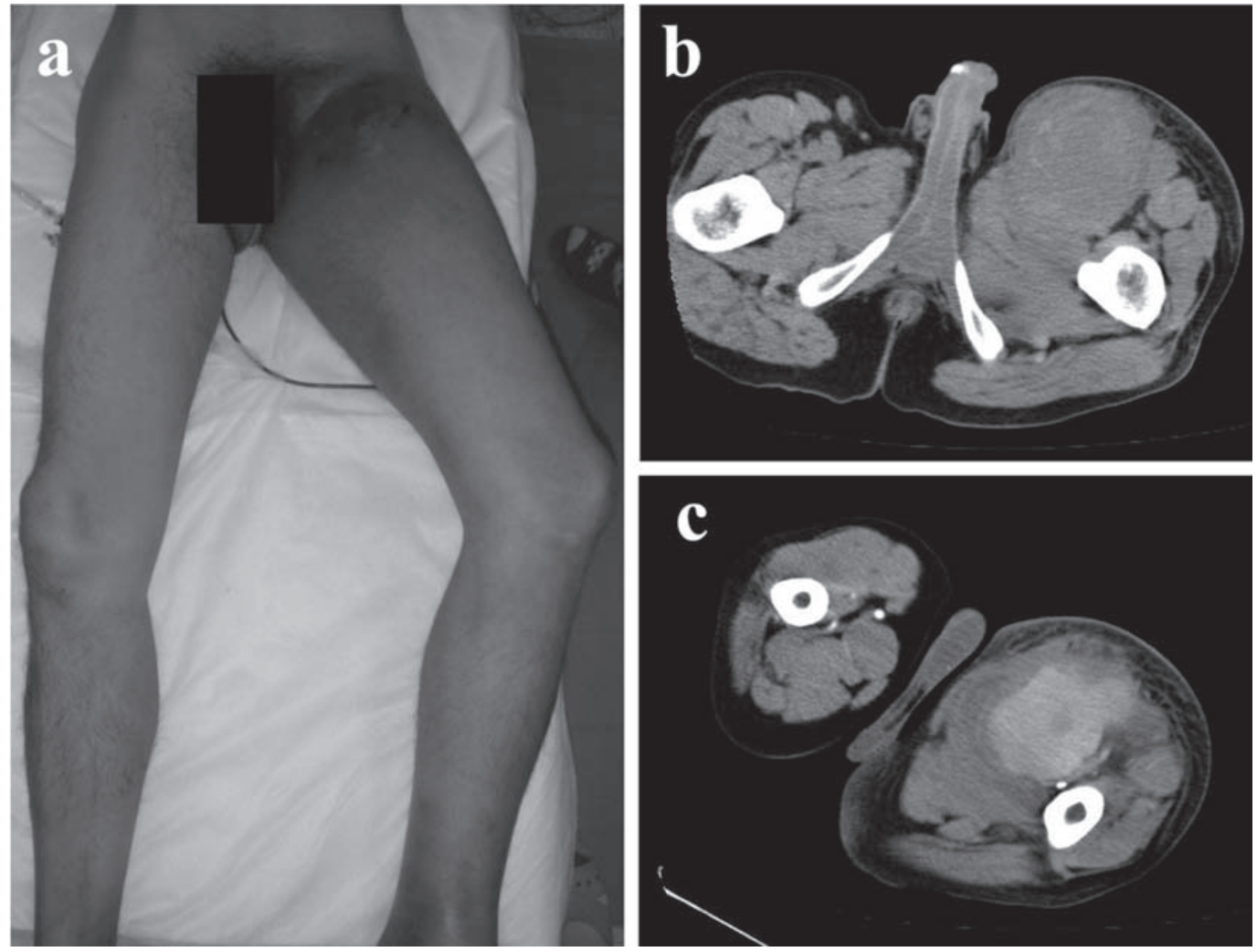

Fig. 1 A: A non-pulsatile mass and entire left lower leg swelling were seen. B: The plain computed tomography scan of the lower leg showed a tumor-like mass about $10 \mathrm{~cm}$ in diameter. $\mathbf{C}$ : Enhanced computed tomography scan of the lower leg showed that the contrast medium leaked out of the left femoral artery and a large pseudoaneurysm was formed in this area.

the lesion.

On admission to our department, his left inguinal mass and left lower limb swelling progressed more than before. Blood examinations revealed a severe inflammatory reaction (WBC: 16,730/ $\mu \mathrm{L}, \mathrm{CRP}: 23.5 \mathrm{mg} / \mathrm{dL}$ ) and a mild hypercoagulable condition (PT: $12.6 \mathrm{sec}$, PT-INR: 1.37, APTT: $39.2 \mathrm{sec}$ ). Before the operation, we performed enhanced CT scan of the lower legs, which showed that the contrast medium leaked out of the left femoral artery, then, a large pseudoaneurysm formed in this area (Fig. 1C). We diagnosed him with left femoral arterial pseudoaneurysm of unknown cause, and decided to perform an emergency operation. Under general anesthesia, after proximal control of the left external iliac artery, we removed a major hematoma through an incision in the groin and found continuous bleeding from a tiny tear in the anterior wall of the left superficial femoral artery (Fig. 2). We closed the tear by uninterrupted suture using 5-0 polypropylene to control the bleeding. Macroscopi-

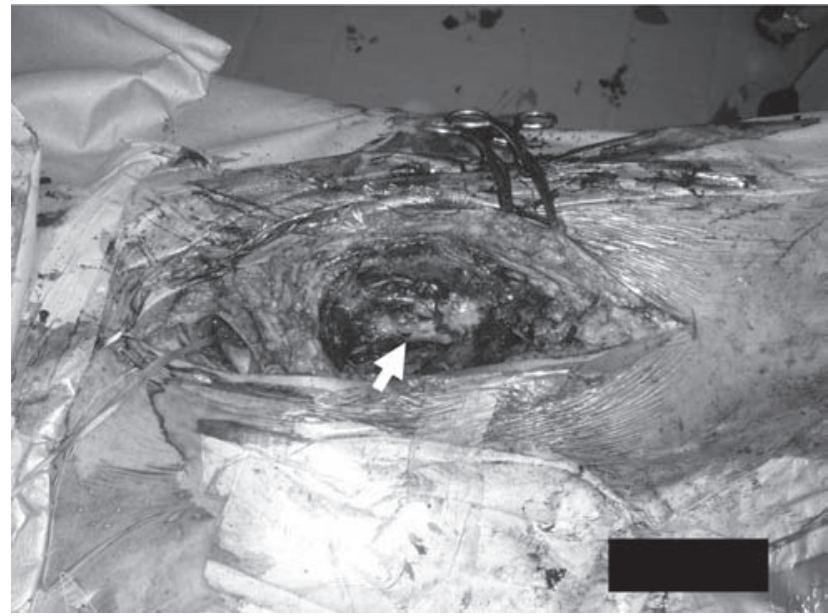

Fig. 2 Intraoperative finding of left femoral area. There was a tiny tear in the anterior wall of the left superficial femoral artery with the removal of a major hematoma (arrow). 


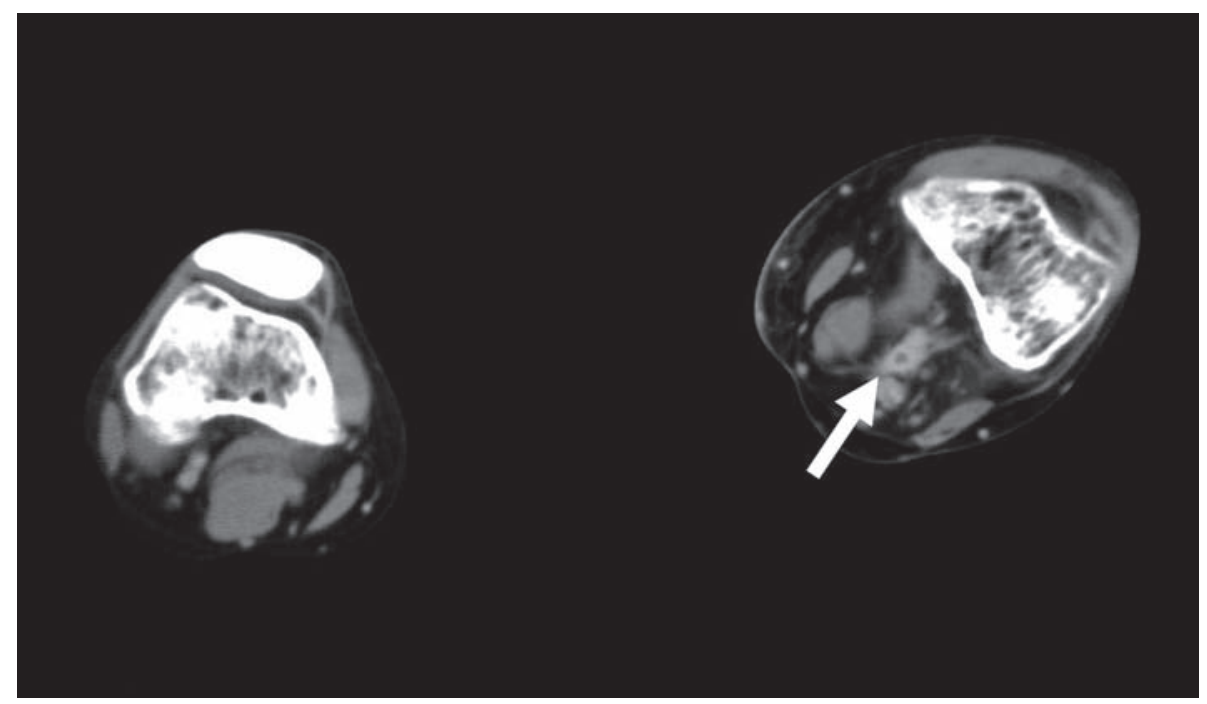

Fig. 3 Enhanced computed tomography scan of the lower leg after seven months postoperatively showed thrombus formation in left popliteal vein (arrow), which was diagnosed as deep venous thrombosis.

cally, the arterial wall condition in this area was normal, with no findings of apparent arteriosclerotic change, true aneurysmal change, or infectious change. Confirming a good blood flow to the distal side of the sutural part by the Doppler flow examination, we finished this operation. After surgery, he experienced rapid resolution of symptoms of left lower leg pain and swelling, and he was discharged without complication on postoperative day 14 .

We suspected that he had a systemic disorder such as connective tissue disease or an autoimmune disease as the background factor of his vascular lesion because he was a young patient. However, as a result of related consultation, uveitis was the only coexisting disease that he had at that time; hence we could not determine the background disease.

After discharge from our hospital, we observed his postoperative clinical course through an outpatient visit once a month. His general status gradually improved, and he remained in stable condition for a while. However, approximately seven months postoperatively, he visited our outpatient department with sudden left lower leg pain, swelling, and reddening. The enhanced CT of the legs showed the findings of venous thrombus of left popliteal vein (Fig. 3). As a result of enhanced CT of the legs, he was diagnosed with deep venous thrombosis. We immediately started anticoagulation treatment with heparin (15,000 U/day for three days) and warfarin (4 mg/day). A couple days later, symptoms such as aphthous stomatitis and pudendal ulcer started to develop progressively, complicating his uveitis. We thought that these were typical symptoms for Behçet's disease, and we made a diagnosis for vasculo-Behçet's disease in conjunction with his vascular lesion based on the Japanese diagnostic criteria for Behçet's disease.

A more detailed and systemic work-up demonstrated that T2-weighted imaging of MRI showed increased signal intensity lesion at the brain-stem area and right optic thalamus without central nervous system (CNS) manifestation, which was a suspected CNS complication, and lower intestinal tract endoscopy showed multiple ulcerous lesions, which was suspected intestinal tract complication. In addition, he was positive for HLA-B51.

Then, he started treatment with oral prednisolone $(1 \mathrm{mg} / \mathrm{kg})$, and his symptoms and the inflammatory reaction on blood examination improved slightly.

\section{Discussion}

BD is a multi-systemic, chronic, inflammatory disorder that is mainly characterized by aphthous stomatitis, genital ulcers, skin lesions and ocular manifestations. In 1937, this disease was first described by Hulusi Behçet in Turkey, and it tended to be frequently seen in the Middle East, Japan, and Mediterranean countries. Today, it has a global distribution, and various reports have been published. This disease has an undulating course with pre- 
cipitating and remission periods. Although several immunological abnormalities have been demonstrated in patients with $\mathrm{BD}$, the detailed pathogenesis still remains unknown.

In addition to the symptoms listed above, sometimes this disease is subject to the development of neurologic, gastrointestinal, or vascular lesions, which is often classified into the specific type of $\mathrm{BD}$, which is distinct from the common type of BD. In general, these particular types of BD have a worse prognosis than the common type because of life-threatening lesions such as irreversible disturbances of the central nervous system, bowel perforations, aneurysm rupture of large vessel, and pulmonary embolism due to deep venous thrombosis. Among these particular types of $\mathrm{BD}, \mathrm{VBD}$, which has been identified in only $7 \%$ to $38 \%$ of BD patients, mainly affects the venous system and central or peripheral arteries. Venous thrombosis, aneurysm formation, and arterial occlusion are well-documented clinical features of VBD. ${ }^{2,3)}$

The diagnosis of BD is based on clinical criteria, which consist of combinations of symptoms because of the lack of universally recognized pathognomonic laboratory tests. ${ }^{1,4)}$ Therefore, it is difficult to diagnose this disease and sometimes a definite diagnosis is made too late. Compared to common symptoms frequently encountered in BD such as aphthous stomatitis or genital ulcers, vascular lesions such as arterial aneurysm or venous thrombosis are rare manifestations, and these often appear during a chronic phase of the disease. Although there are various reports associated with VBD, most are cases that had been diagnosed with BD before the occurrence of vascular lesion. ${ }^{5,6)}$ In our case, the femoral arterial pseudoaneurysm of the leg was the earliest symptoms, and then, deep venous thrombosis and other typical symptoms of BD became apparent. There is no report similar to our case, to our knowledge, and we believe that this clinical course is rare.

Caution should be exercised in patients with a tumor- like mass in the groin. These are often diagnosed as inflammatory lesions or neoplastic lesions from the clinical course or examination findings, in which the correct diagnosis may be difficult to make. Uzun et al. reported that they diagnosed a neck mass with a metastatic tumor and the correct diagnosis of a vascular lesion due to VBD was difficult. ${ }^{7)}$ From our case, we believe it is important to perform enhanced CT or angiography on patients with an inguinal mass with suspicion of vascular lesions such as femoral arterial aneurysm due to VBD.

In conclusion, we experienced a case of femoral arterial pseudoaneurysm and deep venous thrombosis due to VBD with a unique clinical course. To diagnosis this disease definitively, we should consider the possibility of vascular lesions from differential diagnosis with physical symptoms and various examination findings.

\section{REFERENCES}

1) Criteria for diagnosis of Behçet's disease. International Study Group for Behçet's Disease. Lancet. 1990; 335: 1078-80.

2) Koç Y, Güllü I, Akpek G, Akpolat T, Kansu E, Kiraz S, et al. Vascular involvement in Behçet's disease. J Rheumatol. 1992; 19: 402-10.

3) Okamoto H, Sato O. Vasculo- Behçet's disease. Angiology Frontier. 2004; 3: 209-13.

4) Alpsoy E, Zouboulis CC, Ehrlich GE. Mucocutaneous lesions of Behçet's disease. Yonsei Med J. 2007; 48: $573-85$.

5) Dogan SM, Birdane A, Korkmaz C, Ata N, Timuralp B. Right ventricular thrombus with Behçet's syndrome: successful treatment with warfarin and immunosuppressive agents. Tex Heart Inst J. 2007; 34: 360-2.

6) Bardakci H, Kervan U, Boysan E, Birincioglu L, Cobanoglu A. Aortic arch aneurysm, pseudocoarctation, and coronary artery disease in a patient with Behçet's syndrome. Tex Heart Inst J. 2007; 34: 363-5.

7) Uzun L, Ugur MB, Ulukent SC, Ozdemir H, Koca R. Vasculo-Behçet's disease mimicking a metastatic neck mass. Tohoku J Exp Med. 2005; 206: 81-4. 